\title{
Coverage and Area Spectral Efficiency of Clustered Device-to-Device Networks
}

\author{
Mehrnaz Afshang, Harpreet S. Dhillon, and Peter Han Joo Chong
}

\begin{abstract}
This paper develops a new spatial model for deviceto-device (D2D) networks in which the device locations are modeled as a Thomas cluster process. The devices inside a given cluster form D2D links amongst themselves and the direct communication across clusters is not required. This model captures the fact that the devices engaged in D2D communications need to be in close proximity of each other. For this model, we derive easy-to-use expressions for both coverage probability and area spectral efficiency (ASE) assuming that the content of interest is available at a device chosen uniformly at random from the same cluster. One of the important consequences of this analysis is that there exists an optimal number of simultaneously active D2D-Txs that maximizes the ASE. This can be interpreted as the classical tradeoff between more aggressive frequency reuse and higher interference power. Our analysis also provides insights into the effect of scattering variance of each cluster and the density of cluster centers on coverage probability and ASE.
\end{abstract}

Index Terms-Device-to-device (D2D) communication, clustered D2D network, Poisson cluster process, Thomas cluster process, stochastic geometry.

\section{INTRODUCTION}

Enabling direct communication between devices located in close proximity, termed device-to-device (D2D) communications, has several benefits compared to the conventional approach of communicating through a base station in a cellular network [1]. First, the spectral efficiency of the direct link is typically much higher due to smaller link distance. Second, this circumvents the need to establish an end-to-end link through a base station, thereby offloading traffic from cellular networks. Third, while the D2D network can be visualized as an ad hoc network, it incurs a much lower protocol overhead due to the assistance it can get from the cellular network. All these benefits make it an attractive component of both the current $4 \mathrm{G}$ and the future $5 \mathrm{G}$ networks [1], [2].

The D2D communication opens up several exciting possibilities that were otherwise not quite possible with traditional cellular architecture. In particular, devices can now cache popular files that can be shared with the nearby users [3], [4]. We term each such set of proximate users as a cluster. Note that the set of popular files may not be the same across clusters. For instance, a cluster of users sitting in a sports bar will likely be interested in a very different set of files than a cluster of students sitting in a library. In this paper,

M. Afshang is with Wireless@VT, Department of ECE, Virgina Tech, Blacksburg, VA, USA and INFINITUS@NTU, Department of EEE, Nanyang Technological University, Singapore. Email: mehrnaz@vt.edu. H. S. Dhillon is with Wireless@VT, Department of ECE, Virgina Tech, Blacksburg, VA, USA. Email: hdhillon@vt.edu. P. H. J. Chong is with INFINITUS@NTU, Department of EEE, Nanyang Technological University, Singapore. Email: Ehjchong@ntu.edu.sg. we develop a comprehensive spatial model based on Poisson cluster process that accurately captures the clustering of users and the fact that the set of popular files may be different across clusters, thereby limiting the need for D2D communication between devices located across different clusters.

Related work. Powerful tools from stochastic geometry have been successfully applied to the spatial modeling and performance analysis of both wireless ad hoc and cellular networks [5]. More recently, these tools have been used to characterize various aspects of D2D networks, such as mode selection in D2D communication underlaying cellular networks [6], [7], D2D interference management [8], [9], multicast transmissions [10], and distributed caching in D2D networks [11]. The most popular spatial model for D2D networks is the so called Poisson Dipole Process (PDP), where the D2D transmitters are modeled by a homogeneous Poisson Point Process (PPP), with the corresponding receivers located at a predefined fixed distance from each transmitter [8], [9], [12], [13]. While this is a good first-order model, it suffers from two main shortcomings: (i) it does not capture the clustering effect described above, and (ii) the assumption of the constant link distance, which is typically assumed to be the same for all the D2D links, is somewhat restrictive. In this paper we address these shortcomings by developing a new spatial model for D2D networks.

Contributions and outcomes. We focus on the outband D2D communications, in which the D2D and cellular transmissions do not interferer with each other. For this setup, we propose a new and more realistic way of modeling D2D networks in which the devices form clusters instead of being uniformly located on the plane, as was the case in popular models, such as the PDP, discussed above. Furthermore, we assume that the content of interest for a typical device in a given cluster is available at a device chosen uniformly at random in that cluster. This relaxes the fixed link distance assumption described above. Modeling the device locations as a Thomas cluster process [14], we obtain tractable expressions for key performance metrics of interest, such as the outage probability and area spectral efficiency (ASE). One of the important consequences of this analysis is the insights into the "optimal" number of D2D links that should be simultaneously activated in each cluster. More simultaneously active links means potentially higher network throughput, but at the same time it increases interference in the system. We optimize this tradeoff by maximizing ASE over the whole network. 


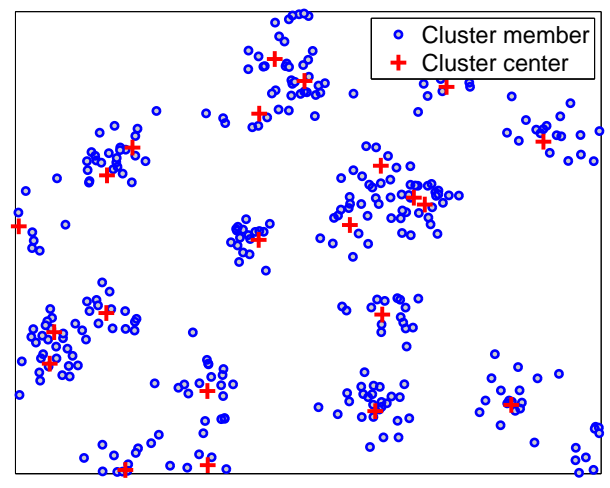

Fig. 1. Illustration of D2D cluster network when cluster members (devices) are normally distributed around cluster center.

\section{SySTEM MODEL}

We consider a D2D network in which the devices exist in physical clusters. We assume that each device has a certain set of files that can be requested by the other devices in the same cluster and the devices across clusters do not communicate. This can be justified in two ways: (i) the inter-cluster distances will be typically much larger than the intra-cluster distances, and (ii) the devices in one cluster may not have information of interest to the devices of the other clusters. The locations of the devices are modeled by a Thomas cluster process [5], [14] in which the cluster centers form a homogeneous PPP $\Phi_{\mathrm{c}}$ with density $\lambda_{\mathrm{c}}$ and the cluster members form finite PPPs around each cluster center $x \in \Phi_{\mathrm{c}}$ with the intensity function

$$
\lambda_{0}\left(y_{x}\right)=\frac{\bar{n}}{2 \pi \sigma^{2}} \exp \left(-\frac{\left\|y_{x}\right\|^{2}}{2 \sigma^{2}}\right),
$$

where $\bar{n}$ is the mean number of points in each cluster. In other words, cluster members (devices) are independent and identically distributed (i.i.d.) according to a symmetric normal distribution with variance $\sigma^{2}$ around each cluster center $x \in \Phi_{\mathrm{c}}$. The devices in the cluster of $x \in \Phi_{\mathrm{c}}$ are denoted by $\mathcal{N}^{x}=\left\{y_{j x}\right\}$. Note that there is no device (almost surely) located at the cluster center. This model is illustrated in Fig. 1. By assuming same mean number of devices $\bar{n}$ for all the clusters, we model the scenario in which the cluster sizes are almost similar but the exact number of devices across clusters are not necessarily the same. We further assume that half of these devices are "potential" D2D transmitters (D2DTxs). The set of simultaneously active D2D-Txs in a cluster is denoted by $\mathcal{B}^{x} \subset \mathcal{N}^{x}$. We assume that the number of simultaneously active D2D-Txs inside each cluster is a Poisson random variable with mean $\bar{m}$.

Without loss of generality, we perform analysis on a typical device, which is a randomly chosen device in a randomly chosen cluster, termed representative cluster, inside the network. Since a representative cluster must have at least one active D2D-Txs for this analysis to make sense, for concreteness we assume that the number of active interferers in that cluster is Poisson distributed with mean $\bar{m}-1$. Due to the stationarity of the process, we assume that $\mathrm{D} 2 \mathrm{D}$ receiver (D2D-Rx) of interest (typical device) is located at the origin and D2D-Txs of cluster $x \in \Phi_{\mathrm{c}}$ are located at $z_{j x}=x+y_{j x}$. Assume representative cluster center to be located at $x_{0} \in \Phi_{\mathrm{c}}$ and D2D-Tx of interest to be at $z_{0 x_{0}}$ (i.e., $j=0$ ), which is at distance $r=\left\|z_{0 x_{0}}\right\|$ away from D2D-Rx of interest. Assuming transmit power of each device to be $P_{\mathrm{d}}$, the received power at D2D-Rx of interest is

$$
P=P_{\mathrm{d}} h_{0 x_{0}}\left\|x_{0}+y_{0 x_{0}}\right\|^{-\alpha},
$$

where $h_{0 x_{0}} \sim \exp (1)$ models Rayleigh fading and $\alpha$ is the path loss exponent. Incorporating shadowing is left as a promising future direction of work. To define interference field, it is useful to first define the set of all simultaneously active D2D-Txs as:

$$
\Psi_{\mathrm{m}}=\cup_{x \in \Phi_{\mathrm{c}}} \mathcal{B}^{x}
$$

where recall that $\mathcal{B}^{x}$ is the set of simultaneously active D2DTxs inside a cluster centered at $x \in \Phi_{\mathrm{c}}$. In this network, the total interference caused at the D2D-Rx of interest can be written as the sum of two independent terms: (i) intra-cluster interference caused by the interfering D2D-Txs inside the representative cluster, and (ii) inter-cluster interference caused by simultaneously active D2D-Txs outside the representative cluster. Recalling that the D2D-Tx of interest is located at $y_{0 x_{0}}$ (i.e., $j=0$ ) with respect to the cluster center $x_{0}$, the intra-cluster interference power can be expressed as:

$$
I_{\mathrm{Tx}-\text { cluster }}=\sum_{j \in \mathcal{B}^{x_{0}} \backslash 0} P_{\mathrm{d}} h_{j x_{0}}\left\|x_{0}+y_{j x_{0}}\right\|^{-\alpha} .
$$

Similarly, the interference from the simultaneously active D2D-Txs outside the representative cluster, $x_{0}$, at the D2D-Rx of interest can be expressed as:

$$
I_{\Psi_{\mathrm{m}} \backslash \mathrm{Tx} \text {-cluster }}=\sum_{x \in \Phi_{\mathrm{c}} \backslash x_{0}} \sum_{j \in \mathcal{B}^{x}} P_{\mathrm{d}} h_{j x}\left\|x+y_{j x}\right\|^{-\alpha} .
$$

The total clustered network interference is sum of the intracluster interference and the inter-cluster interference:

$$
I_{\Psi_{\mathrm{m}}}=I_{\Psi_{\mathrm{m}} \backslash \mathrm{Tx}-\text { cluster }}+I_{\mathrm{Tx}-\text { cluster }} .
$$

Now, recalling that the distance between the typical device and the D2D-Tx of interest is $r=\left\|z_{0 x_{0}}\right\|$, the SIR experienced by the typical device is

$$
\operatorname{SIR}(r)=\frac{P_{\mathrm{d}} h_{0 x_{0}} r^{-\alpha}}{I_{\Psi_{\mathrm{m}} \backslash \mathrm{Tx} \text {-cluster }}+I_{\mathrm{Tx}-\text { cluster }}} .
$$

For notational simplicity, we assume that the system operates in the interference limited regime, i.e., the background noise is negligible compared to the interference and is hence ignored. This means that the transmit power term cancels in the SIR expression above and can hence be ignored, i.e., we can set $P_{\mathrm{d}}=1$ without any loss of generality.

\section{Clunstered D2D Network Analysis}

This is the main technical section of the paper. We begin by deriving the Laplace transform of the interference powers, using which we will derive the coverage probability of the typical device and the ASE of the whole network. 


\section{A. Laplace Transform of Interference}

In this subsection, we derive the Laplace transforms of intracluster and inter-cluster interference powers. It is important to note that a similar analysis was performed for the Thomas cluster process in [14], albeit for a different setup. In [14], the receiver is not a part of the cluster process and the serving distance is fixed. In this paper, we generalize both these assumptions by sampling the receiver from the cluster process itself. We also use a key characteristic of Rician distribution to provide much more tractable approach, which can also be used to provide simpler expressions for the setup considered in [14].

1) Laplace transform of intra-cluster interference: Before performing the intra-cluster interference analysis, we derive the density function of the distances from simultaneously active D2D-Txs $\mathcal{B}^{x_{0}}$ located at $\left\{z_{j x_{0}}\right\}$ inside the representative cluster to the D2D-Rx of interest in the following Lemma.

Lemma 1. Assuming representative cluster center is located at $x_{0}$, the probability density function of

1) The distance from the intra-cluster interferers to the typical device, i.e., $w=\left\|x_{0}+y_{j x_{0}}\right\|, j \neq 0$, is

$$
f_{W}\left(w \mid \nu_{0}\right)=\frac{w}{\sigma^{2}} \exp \left(-\frac{w^{2}+\nu_{0}^{2}}{2 \sigma^{2}}\right) I_{0}\left(\frac{w \nu_{0}}{\sigma^{2}}\right),
$$

2) The distance from the D2D-Tx of interest to the typical device, i.e., $r=\left\|x_{0}+y_{j x_{0}}\right\|, j=0$, is

$$
f_{R}\left(r \mid \nu_{0}\right)=\frac{r}{\sigma^{2}} \exp \left(-\frac{r^{2}+\nu_{0}^{2}}{2 \sigma^{2}}\right) I_{0}\left(\frac{r \nu_{0}}{\sigma^{2}}\right),
$$

where $\nu_{0}=\left\|x_{0}\right\|$, and $I_{0}($.$) is the modified Bessel function$ with order zero.

Proof. Note that the intra-cluster distances $\left\|x_{0}+y_{j x_{0}}\right\|$ are i.i.d. when conditioned on the common distance from the representative cluster to the typical device, $\nu_{0}=\left\|x_{0}\right\|$. Since $y_{j x_{0}}$ is zero-mean Gaussian random variable in $\mathbb{R}^{2}$, the distances are all Rician distributed when conditioned on $\nu_{0}$.

We now derive the Laplace transform of the received power from intra-cluster interferers at the typical device.

Lemma 2. Laplace transform of the intra-cluster interference power given by (4) conditioned on the distance $\nu_{0}=\left\|x_{0}\right\|$ between the cluster center and the typical device is given by

$$
\begin{aligned}
& \mathcal{L}_{I_{\mathrm{Tx}-\text { cluster }}}\left(s \mid \nu_{0}\right)= \\
& \quad \exp \left(-(\bar{m}-1) \int_{0}^{\infty} \frac{s w^{-\alpha}}{1+s w^{-\alpha}} f_{W}\left(w \mid \nu_{0}\right) \mathrm{d} w\right),
\end{aligned}
$$

where $f_{W}\left(w \mid \nu_{0}\right)=\frac{w}{\sigma^{2}} \exp \left(-\frac{w^{2}+\nu_{0}^{2}}{2 \sigma^{2}}\right) I_{0}\left(\frac{w \nu_{0}}{\sigma^{2}}\right), \quad w>0$.

Proof. See Appendix A.

A closed form lower bound for Laplace transform of intracluster can be obtained by using Jensen's inequality. The result is given in the following Corollary.

Corollary 1. The lower bound on Laplace transform of intracluster interference at the D2D-Rx of interest is given by:

$$
\mathcal{L}_{I_{\mathrm{Tx}-\text { cluster }}}(s) \geq \exp \left(-\pi \frac{(\bar{m}-1)}{4 \sigma^{2}} s^{\frac{2}{\alpha}} C(\alpha)\right),
$$

where $C(\alpha)=\frac{2 \pi / \alpha}{\sin (2 \pi / \alpha)}$.

Proof. Due to lack of space, the proof is delegated to the extended version of this paper [15].

We will use this lower bound to derive closed form approximations for coverage probability and ASE, which will lead to several system design guidelines later in this section.

2) Laplace transform of inter-cluster interference: Similar to the intra-cluster analysis, we derive the probability density function of the distances from simultaneously active D2D-Txs outside the representative cluster $\Psi_{\mathrm{m}}=\cup_{x \in \Phi_{\mathrm{c}} \backslash x_{0}} \mathcal{B}^{x}$ located at $\left\{z_{j x}\right\}$ to the D2D-Rx of interest in the following Lemma.

Lemma 3. The probability density function of the distances from the intra-cluster interferers to the D2D-Rx of interest, $u=\left\|x+y_{j x}\right\|$, is given by:

$$
f_{U}(u \mid \nu)=\frac{u}{\sigma^{2}} \exp \left(-\frac{u^{2}+\nu^{2}}{2 \sigma^{2}}\right) I_{0}\left(\frac{u \nu}{\sigma^{2}}\right),
$$

where $\nu=\|x\|, u>0$, and $I_{0}($.$) is the modified Bessel$ function with order zero.

Proof. The proof follows on the same lines as Lemma 1.

The Laplace transform of inter-cluster interference at the typical device is derived next.

Lemma 4. The Laplace transform of inter-cluster interference at D2D-Rx of interest in (5) is given by $\mathcal{L}_{I_{\Psi_{\mathrm{m}} \backslash \mathrm{Tx}-\text { cluster }}}(s)=$

$$
\begin{aligned}
\exp (-2 & \lambda_{\mathrm{c}} \int_{0}^{\infty}\left(1-\exp \left(-\bar{m} \int_{0}^{\infty} \frac{s u^{-\alpha}}{1+s u^{-\alpha}}\right.\right. \\
& \left.\left.\left.\times f_{U}(u \mid \nu) \mathrm{d} u\right)\right) \nu \mathrm{d} \nu\right)
\end{aligned}
$$

where $f_{U}(u \mid \nu)=\frac{u}{\sigma^{2}} \exp \left(-\frac{u^{2}+\nu^{2}}{2 \sigma^{2}}\right) I_{0}\left(\frac{u \nu}{\sigma^{2}}\right) \quad u>0$.

Proof. See Appendix B.

Using Taylor expansion of the exponential function in the result in Lemma 4, we can obtain a closed form lower bound on the Laplace transform. The result is given next.

Corollary 2. Laplace transform of inter-cluster interference is lower bounded by:

$$
\mathcal{L}_{I_{\Psi_{\mathrm{m}} \backslash \mathrm{Tx}-\text { cluster }}}(s) \geq \exp \left(-\pi \lambda_{\mathrm{c}} \bar{m} s^{2 / \alpha} C(\alpha)\right),
$$

where $C(\alpha)=\frac{2 \pi / \alpha}{\sin (2 \pi / \alpha)}$.

Proof. See Appendix C.

This lower bound will be useful in the coverage probability analysis to gain insights into several system design guidelines.

\section{B. Coverage Probability of the Typical Device}

We begin this subsection by formally defining the SIR coverage probability for the link of interest.

Definition 1. (SIR coverage probability). It is the probability that the received SIR at the D2D-Rx of interest exceeds a pre-defined threshold, expressed mathematically as

$$
\mathrm{P}_{\mathrm{c}}=\mathbb{E}_{R}[\mathbb{P}\{\operatorname{SIR}(R)>\beta \mid R\}],
$$


where $\beta$ is the threshold for successful demodulation and decoding at the receiver.

Now, the coverage probability for D2D-Rx of interest is derived by the following Theorem.

Theorem 1. Using the Laplace transforms of interference in (9) and (12), the coverage probability of the D2D-Rx of interest is given by:

$$
\begin{aligned}
\mathrm{P}_{\mathrm{c}}=\int_{0}^{\infty} \int_{0}^{\infty} & \mathcal{L}_{I_{\psi_{\mathrm{m}} \backslash \mathrm{Tx}-\text { cluster }}}\left(\beta r^{\alpha}\right) \mathcal{L}_{I_{\mathrm{Tx}-\text { cluster }}}\left(\beta r^{\alpha} \mid \nu_{0}\right) \\
& f_{R}\left(r \mid \nu_{0}\right) f_{V_{0}}\left(\nu_{0}\right) \mathrm{d} r \mathrm{~d} \nu_{0},
\end{aligned}
$$

where $f_{V_{0}}\left(\nu_{0}\right)=\frac{\nu_{0}}{\sigma^{2}} \exp \left(-\frac{\nu_{0}^{2}}{2 \sigma^{2}}\right), \quad \nu_{0}>0$.

Proof. From the definition of coverage probability, we have

$$
\begin{aligned}
& \mathrm{P}_{\mathrm{c}}=\mathbb{E}_{R}\left[\mathbb{P}\left\{\frac{h_{0 x_{0}} r^{-\alpha}}{I_{\Psi_{\mathrm{m}} \backslash \mathrm{Tx}-\text { cluster }}+I_{\mathrm{Tx}-\text { cluster }}}>\beta \mid R\right\}\right] \\
& =\mathbb{E}_{R}\left[\mathbb{P}\left\{h_{0 x_{0}}>\operatorname{Tr}^{\alpha}\left(I_{\Psi_{\mathrm{m}} \backslash \mathrm{Tx} \text {-cluster }}+I_{\mathrm{Tx} \text {-cluster }}\right) \mid R\right\}\right] \\
& \stackrel{(a)}{=} \mathbb{E}_{R} \mathbb{E}\left[\exp \left(-\operatorname{Tr}^{\alpha}\left(I_{\Psi_{\mathrm{m}} \backslash \mathrm{Tx} \text {-cluster }}+I_{\mathrm{Tx} \text {-cluster }}\right)\right) \mid R\right],
\end{aligned}
$$

where $(a)$ follows from Rayleigh fading assumption. The result now follows from the independence of intra-cluster and inter-cluster interference powers, followed by de-conditioning over $R$ given $\nu_{0}$ using the serving link distribution given by (8), followed by de-conditioning over $\nu_{0}$, which is simply a Rayleigh distributed random variable due to the position being sampled from a Gaussian distribution in $\mathbb{R}^{2}$.

\section{Area Spectral Efficiency}

The ASE simply denotes the average number of bits transmitted per unit time per unit bandwidth per unit area. Assuming that all the D2D-Txs use Gaussian codebooks for their transmissions, we can use Shannon's capacity formula to define ASE $=\lambda \log _{2}(1+\beta) \mathrm{P}_{\mathrm{c}}$, where $\lambda$ is the density of the active transmitters and $P_{c}$ is the coverage probability of the typical node. This definition can be easily specialized to our setup. The result is given in the following Proposition.

Proposition 1. The ASE of the clustered D2D network is

$$
\mathrm{ASE}=\bar{m} \lambda_{\mathrm{c}} \log _{2}(1+\beta) \mathrm{P}_{\mathrm{c}},
$$

where $\mathrm{P}_{\mathrm{c}}$ is given by (15) and $\bar{m} \lambda_{\mathrm{c}}$ represents the average density of simultaneously active D2D-Txs inside the network.

\section{Key System Design Guidelines}

In this subsection, we gain important system design insights. We first derive closed-form approximations for the coverage probability and ASE in the following corollaries.

Corollary 3. The closed form approximation for the coverage probability of a typical device is

$$
\mathrm{P}_{\mathrm{c}} \approx \frac{1}{\left(4 \pi \lambda_{\mathrm{c}} \sigma^{2} \bar{m}+\bar{m}-1\right) \beta^{\frac{2}{\alpha}} C(\alpha)+1},
$$

where $C(\alpha)=\frac{2 \pi / \alpha}{\sin (2 \pi / \alpha)}$.

Proof. The proof follows from the expectation of the lower bounds provided in (10) and (13) when $s=\beta r^{\alpha}$, with

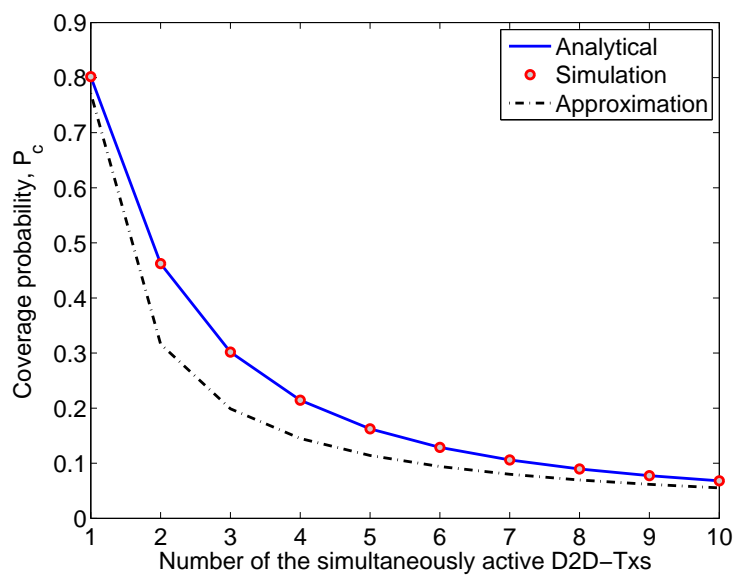

Fig. 2. Coverage probability versus number of simultaneously active D2DTxs when $\sigma=10$ and $\lambda_{\mathrm{c}}=150$ clusters $/ \mathrm{km}^{2}$.

respect to the marginal distribution of serving link distance $f_{R}(r)=\frac{r}{2 \sigma^{2}} \exp \left(-\frac{r^{2}}{4 \sigma^{2}}\right)$. This is an approximation because of independent de-conditioning over serving link and intracluster interferer distance distributions.

Corollary 4. The closed form approximation for ASE is

$$
\mathrm{ASE} \approx \frac{\bar{m} \lambda_{\mathrm{c}}}{\left(4 \pi \lambda_{\mathrm{c}} \sigma^{2} \bar{m}+\bar{m}-1\right) \beta^{\frac{2}{\alpha}} C(\alpha)+1},
$$

Proof. The proof simply follows from the definition of the ASE when $P_{c}$ is substituted by (17).

From these closed form bounds, two remarks are in order.

Remark 1 (Scattering variance, $\sigma^{2}$ ). The approximations corresponding to the ASE and $\mathrm{P}_{\mathrm{c}}$ are decreasing functions of $\sigma^{2}$. This observation shows that the coverage probability and ASE will increase if the devices form more dense clusters around cluster centers, as opposed to more spread-out clusters. While this observation is based on the approximation, we will validate it in the next section.

Remark 2 (Cluster center density, $\lambda_{\mathrm{c}}$ ). Increasing cluster center density $\lambda_{\mathrm{c}}$ has a conflicting effect on the approximations of coverage and ASE: coverage decreases and ASE increases. This shows that more and more clusters can be accommodated as long as the coverage probability remains acceptable. This insight has been validated through numerical results in the extended version of this paper [15].

We now comment on the optimum number of D2D links that should be activated to maximize ASE. Recall that while more active links means potentially higher ASE, it also increases interference significantly. To gain more insights into the number of links that should be activated simultaneously in each cluster, we define ASE optimization problem as:

$$
\mathrm{ASE}^{*}=\max _{\bar{m} \in 1, \ldots, N / 2} \bar{m} \lambda_{\mathrm{c}} \log _{2}(1+\beta) \mathrm{P}_{\mathrm{c}} .
$$

We will revisit the tradeoff over the number of simultaneously active links in subsection IV-B. By solving the ASE optimization problem numerically, we demonstrate the existence of an optimal value of $\bar{m}$ that maximizes the ASE. 


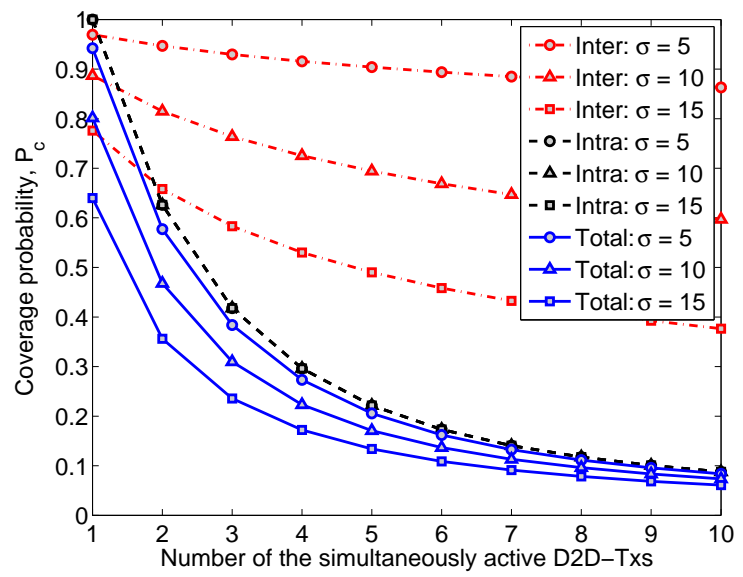

Fig. 3. Coverage probability versus number of simultaneously active D2DTxs for $\lambda_{\mathrm{c}}=150$ clusters $/ \mathrm{km}^{2}$ and different value of $\sigma$.

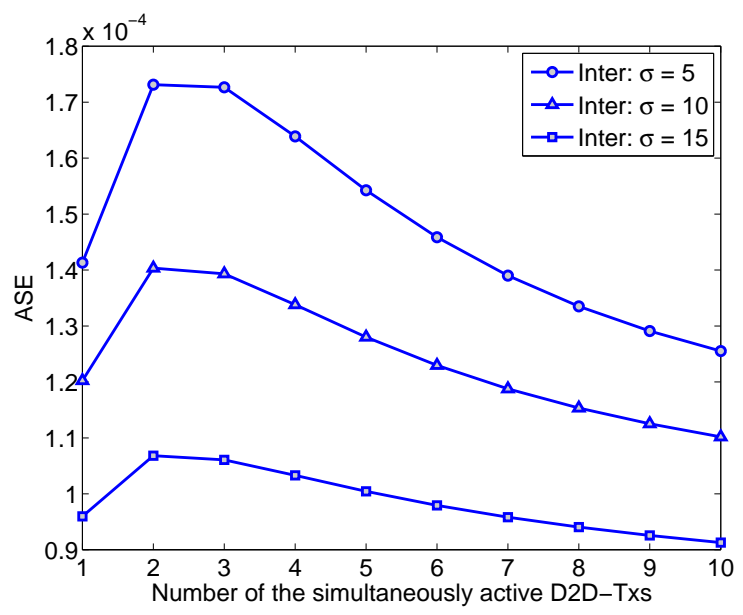

Fig. 4. ASE versus number of simultaneously active D2D-Txs for $\lambda_{\mathrm{c}}=150$ clusters $/ \mathrm{km}^{2}$ and different value of $\sigma$.

\section{RESUlts AND Discussion}

\section{A. Validation of results}

In this subsection, we validate the analytical results and investigate the tightness of the closed form approximation for coverage probability derived in the previous section. In all simulations, the locations of devices are drawn from a Thomas cluster process defined over a square of area $50 \times 50 \mathrm{~km}^{2}$. In this setup, the center of clusters are spatially distributed as PPP, and the devices are normally scattered around them. We set the SIR threshold for the successful demodulation and decoding, $\beta$, as $0 \mathrm{~dB}$. Comparing the analytical and simulation results in Fig. 2, we note that they are a perfect match, thereby confirming the accuracy of the analysis.

\section{B. Optimum Number of Simultaneously Active Links}

We plot the coverage probability in the presence of (i) only inter-cluster interference, (ii) intra-cluster interference, and (iii) total interference in Fig. 3. It is easy to infer that in the proposed clustered network, coverage probability of a typical device is highly influenced by the intra-cluster interference.
Interestingly, the coverage probability in the absence of intercluster interference is independent of the scattering variance. This is because scattering variance has two counter-balancing effects that cancel each other exactly: decreasing scattering variance increases intra-cluster interference by reducing the link distances to the interfering devices, and improves the desired link quality by again reducing the distance to the serving device. For coverage probability in the absence of the intracluster interference, it can be seen that the smaller scattering variance provides higher coverage probability, which is also true for the coverage probability computed in the presence of both inter- and intra-cluster interference.

Fig. 4 presents ASE with respect to the number of simultaneously active D2D-Txs. While more simultaneously active links may improve ASE, they may also increase interference significantly. Interestingly, it can be seen that the optimal value of ASE is the same for various scattering variances. This is because, intra-cluster interference has dominant impact on this tradeoff compared to the inter-cluster interference. Moreover, recall that the coverage probability in the absence of the intercluster interference is independent of the scattering variance which leads to the same optimal value for a range of scattering variances. It can also be seen that small scattering variances result in higher ASE which highlights the importance of the short range D2D communication.

\section{CONCLUSION}

In this paper, we have developed a comprehensive framework to study proximity-based D2D communication. By modeling the D2D network as a Poisson cluster process, we capture the fact that devices engaging in D2D communication are in close proximity to each other. Using tools from stochastic geometry, we have studied the network performance in terms of coverage probability of the typical device and area spectral efficiency of the whole network when the content of interest is available inside the cluster at random. To the best of our knowledge, this is the first stochastic geometry-based analysis of clustered D2D networks. An important consequence of our results is the optimal number of D2D links that should be activated in each cluster. We also provided a closed-form approximation for coverage probability, using which a closedform approximation for ASE was derived. These results showed that increasing the density of simultaneously active cluster always increases ASE. Hence, cluster center density can be increased as long as coverage probability remains acceptable. The baseline clustered D2D network can be easily extended to characterize several aspects of current and future D2D networks, such as: (i) smart content placement, where content of interest is available at the $k$-closest device to the D2D-Rx of interest, and (ii) inband D2D communication, where devices reuse the spectrum used by the cellular system [16]. The framework should also be extended to other cluster processes, such as the Matern cluster process.

\section{APPENDIX}

\section{A. Proof of Lemma 2}

Recall that SIR is independent of the $P_{\mathrm{d}}$ in our setup, therefore we had set $P_{\mathrm{d}}=1$ in (4). The Laplace transform of 
intra-cluster aggregate interference caused at the D2D-Rx of interest located at the representative cluster center is given by

$$
\begin{aligned}
& \mathcal{L}_{\mathrm{I}_{\mathrm{TX} \text {-cluster }}}\left(s \mid \nu_{0}\right)=\mathbb{E}\left[\exp \left(-s I_{\mathrm{TX}-\text { cluster }}\right)\right] \\
& =\mathbb{E}\left[\exp \left(-s \sum_{j \in \mathcal{B}^{x_{0}} \backslash 0} h_{j x_{0}}\left\|y_{j x_{0}}+x_{0}\right\|^{-\alpha}\right)\right] \\
& =\mathbb{E}_{\mathcal{B}^{x_{0}}}\left[\prod_{j \in \mathcal{B}^{x_{0}} \backslash 0} \mathbb{E}_{h_{j x_{0}}}\left[\exp \left(-s h_{j x_{0}}\left\|y_{j x_{0}}+x_{0}\right\|^{-\alpha}\right)\right]\right] \\
& \stackrel{(a)}{=} \mathbb{E}_{\mathcal{B}^{x_{0}}}\left[\prod_{j \in \mathcal{B}^{x_{0}} \backslash 0} \frac{1}{1+s\left\|y_{j x_{0}}+x_{0}\right\|^{-\alpha}}\right] \\
& \stackrel{(b)}{=} \exp \left(-(\bar{m}-1) \int_{\mathbb{R}^{2}} \frac{s\left\|y_{j x_{0}}+x_{0}\right\|^{-\alpha}}{1+s\left\|y_{j x_{0}}+x_{0}\right\|^{-\alpha}} f_{Y}\left(y_{j x_{0}}\right) \mathrm{d} y_{j x_{0}}\right) \\
& \stackrel{(c)}{=} \exp \left(-(\bar{m}-1) \int_{0}^{\infty} \frac{s w^{-\alpha}}{1+s w^{-\alpha}} f_{W}\left(w \mid \nu_{0}\right) \mathrm{d} w\right),
\end{aligned}
$$

where (a) follows from the definition of Laplace transform and the fact that $h_{j x_{0}} \sim \exp (1)$, (b) follows from the fact that the locations of intra-cluster devices conditioned on $x_{0} \in \Phi_{\mathrm{c}}$ are independent. The result followed by expectation over the relative location of devices to cluster center, $y_{j x_{0}}$, where $f_{Y}\left(y_{j x_{0}}\right)=\frac{1}{2 \pi \sigma^{2}} \exp \left(-\frac{\left\|y_{j x_{0}}\right\|^{2}}{2 \sigma^{2}}\right)$ and using the definition of probability generating function of Poisson distribution where the $\bar{m}-1$ is the average number of intra-cluster interfering devices per cluster, and (c) follows from converting coordinates from Cartesian to polar and some algebraic manipulation where $f_{W}\left(w \mid \nu_{0}\right)$ denotes the distribution of the intra-cluster distance density function given by (7).

\section{B. Proof of Lemma 4}

The Laplace transform of the aggregate interference from the inter-cluster interferers at the D2D-Rx of interest located at the origin is

$$
\begin{aligned}
& \mathcal{L}_{I_{\Psi_{\mathrm{m}} \backslash \mathrm{Tx} \text {-cluster }}}(s)=\mathbb{E}\left[\exp \left(-s I_{\Psi_{\mathrm{m}} \backslash \mathrm{Tx} \text {-cluster }}\right)\right] \\
& =\mathbb{E}\left[\exp \left(-s \sum_{x \in \Phi_{\mathrm{c}} \backslash x_{0}} \sum_{j \in \mathcal{B}^{x}} h_{j x}\left\|x+y_{j x}\right\|^{-\alpha}\right)\right] \\
& =\mathbb{E}_{\Phi_{\mathrm{c}}} \prod_{x \in \Phi_{\mathrm{c}} \backslash x_{0}} \mathbb{E}_{\mathcal{B}^{x}}\left[\prod_{j \in \mathcal{B}^{x}} \mathbb{E}_{h_{j x}}\left[\exp \left(-s h_{j x}\left\|x+y_{j x}\right\|^{-\alpha}\right)\right]\right] \\
& \stackrel{(a)}{=} \mathbb{E}_{\Phi_{\mathrm{c}}}\left[\prod_{x \in \Phi_{\mathrm{c}} \backslash x_{0}} \mathbb{E}_{\mathcal{B}^{x}}\left[\prod_{j \in \mathcal{B}^{x}} \frac{1}{1+s\left\|x+y_{j x}\right\|^{-\alpha}}\right]\right] \\
& \stackrel{(b)}{=} \mathbb{E}_{\Phi_{\mathrm{c}}}\left[\prod_{x \in \Phi_{\mathrm{c}}} \exp \left(\int_{0}^{\infty}-\bar{m} \frac{s u^{-\alpha}}{1+s u^{-\alpha}} f_{U}(u \mid v) \mathrm{d} u\right)\right] \\
& \stackrel{(c)}{=} \exp \left(-2 \pi \lambda_{\mathrm{c}} \int_{0}^{\infty}\left(1-\exp \left(-\bar{m} \int_{0}^{\infty} \frac{s u^{-\alpha}}{1+s u^{-\alpha}}\right.\right.\right. \\
& \left.\left.\left.\quad \times f_{U}(u \mid \nu) \mathrm{d} u\right)\right) \nu \mathrm{d} \nu\right),
\end{aligned}
$$

where (a) arises from the definition of Laplace transform and the fact that $h_{j x} \sim \exp (1)$, and (b) follows from the fact that distances from inter-cluster interfering devices to the typical device $u=\left\|y_{j x}+x\right\|$, conditioned on the $\nu=\|x\|$, are i.i.d., followed by the application of probability generating function of Poisson distribution and density function of intercluster distances given by (11). Finally (c) follows from the probability generating functional (PGFL) of PPP [5].

\section{Proof of Corollary 2}

The lower bound can be derived as follows:

$$
\begin{aligned}
& \mathcal{L}_{I_{\Psi_{\mathrm{m}} \backslash \mathrm{Tx} \text {-cluster }}}(s) \\
\stackrel{(a)}{\geq} & \exp \left(-2 \pi \lambda_{\mathrm{c}} \int_{0}^{\infty}\left(\bar{m} \int_{0}^{\infty} \frac{s u^{-\alpha}}{1+s u^{-\alpha}} f_{U}(u \mid \nu) \mathrm{d} u \nu \mathrm{d} \nu\right)\right) \\
\stackrel{(b)}{=} & \exp \left(-2 \pi \lambda_{\mathrm{c}}\left(\bar{m} \int_{0}^{\infty} \frac{s u^{-\alpha}}{1+s u^{-\alpha}} u \mathrm{~d} u\right)\right) \\
\stackrel{(c)}{=} & \exp \left(-\pi \lambda_{\mathrm{c}}\left(\bar{m} s^{2 / \alpha} \int_{0}^{\infty} \frac{\mathrm{d} z}{1+z^{\alpha / 2}}\right)\right) \\
= & \exp \left(-\pi \lambda_{\mathrm{c}}\left(\bar{m} s^{2 / \alpha} C(\alpha)\right)\right)
\end{aligned}
$$

where (a) follows from the exponential Taylor series expansion and the fact that $1-\exp (-a x) \leq a, a \geq 0$, and (b) follows from the Rician distribution property that $\int_{0}^{\infty} f_{U}(u \mid \nu) \nu \mathrm{d} \nu=$ $u$, where $f_{U}(u \mid \nu)$ is given by (11).

\section{REFERENCES}

[1] K. Doppler, M. Rinne, C. Wijting, C. B. Ribeiro, and K. Hugl, "Deviceto-device communication as an underlay to LTE-advanced networks," IEEE Commun. Magazine, vol. 47, no. 12, pp. 42-49, Dec. 2009.

[2] X. Lin, J. G. Andrews, A. Ghosh, and R. Ratasuk, "An overview of 3GPP device-to-device proximity services," IEEE Commun. Magazine, vol. 52, no. 4, pp. 40-48, Apr. 2014.

[3] N. Golrezaei, P. Mansourifard, A. Molisch, and A. Dimakis, "Basestation assisted device-to-device communications for high-throughput wireless video networks," IEEE Trans. on Wireless Commun., vol. 13, no. 7, pp. 3665-3676, Jul. 2014.

[4] Y. Zhang, E. Pan, L. Song, W. Saad, Z. Dawy, and Z. Han, "Social network aware device-to-device communication in wireless networks," IEEE Trans. on Wireless Commun., vol. 14, no. 1, pp. 177-190, Jan. 2015.

[5] M. Haenggi, Stochastic Geometry for Wireless Networks. Cambridge University Press, 2012.

[6] X. Lin, J. G. Andrews, and A. Ghosh, "Spectrum sharing for device-todevice communication in cellular networks," IEEE Trans. on Wireless Commun., vol. 13, no. 12, Dec. 2014.

[7] H. ElSawy and E. Hossain, "Analytical modeling of mode selection and power control for underlay D2D communication in cellular networks," IEEE Trans. on Commun., vol. 62, no. 11, pp. 4147-4161, Nov. 2014.

[8] H. Sun, M. Wildemeersch, M. Sheng, and T. Q. Quek, "D2D enhanced heterogeneous cellular networks with dynamic TDD," to appear, IEEE Trans. on Wireless Commun., 2015, available online: arxiv.org/abs/1406.2752.

[9] A. H. Sakr and E. Hossain, "Cognitive and energy harvesting-based D2D communication in cellular networks: Stochastic geometry modeling and analysis," IEEE Trans. on Commun., vol. 63, no. 5, pp. 1867-1880, May. 2015.

[10] X. Lin, R. Ratasuk, A. Ghosh, and J. G. Andrews, "Modeling, analysis and optimization of multicast device-to-device transmissions," IEEE Trans. on Wireless Commun., vol. 13, no. 8, pp. 4346-4359, Aug. 2014.

[11] S. Krishnan and H. S. Dhillon, "Distributed caching in device-todevice networks: A stochastic geometry perspective," to appear in Proc. Asilomar, Pacific Grove, CA, Nov. 2015.

[12] H. Feng, H. Wang, X. Xu, and C. Xing, "A tractable model for deviceto-device communication underlaying multi-cell cellular networks," in Proc., IEEE Intl. Conf. on Commun. (ICC), Jun. 2014.

[13] G. George, R. K. Mungara, and A. Lozano, "An analytical framework for device-to-device communication in cellular networks," 2014, available online: arxiv.org/abs/1407.2201.

[14] R. K. Ganti and M. Haenggi, "Interference and outage in clustered wireless ad hoc networks," IEEE Trans. on Info. Theory, vol. 55, no. 9, pp. 4067-4086, Sep. 2009.

[15] M. Afshang, H. S. Dhillon, and P. H. J. Chong, "Modeling and performance analysis of clustered device-to-device networks," submitted to IEEE Trans. on Wireless Commun., available online: arxiv.org/abs/1508.02668.

[16] M. Afshang and H. S. Dhillon, "Spatial modeling of device-to-device networks: Poisson cluster process meets Poisson hole process," to appear in Proc. Asilomar, Pacific Grove, CA, Nov. 2015. 